\title{
Quasispectra of solvable Lie algebra homomorphisms into Banach algebras
}

\author{
by
}

Anar Dosiev (Ankara)

\begin{abstract}
We propose a noncommutative holomorphic functional calculus on absolutely convex domains for a Banach algebra homomorphism $\pi$ of a finite-dimensional solvable Lie algebra $\mathfrak{g}$ in terms of quasispectra $\sigma(\pi)$. In particular, we prove that the joint spectral radius of a compact subset in a solvable operator Lie subalgebra coincides with the geometric spectral radius with respect to a quasispectrum.
\end{abstract}

1. Introduction. One of the central topics of modern analysis is the concept of a function in several noncommuting variables or more generally a noncommutative function algebra. A motivation for such noncommutative generalization is provided, for instance, by the functional calculus problem for a family of noncommuting operators. The aim of a functional calculus is to obtain an operator realization of a (noncommutative) function algebra. More precisely, if there is a continuous algebra homomorphism from a noncommutative function algebra $\mathcal{A}$ into the Banach algebra $\mathcal{B}(X)$ of all bounded linear operators on a complex Banach space $X$, such that the noncommuting variables in $\mathcal{A}$ can be sent to a given operator family in $\mathcal{B}(X)$, then we say that there is a functional calculus with functions from $\mathcal{A}$ in $\mathcal{B}(X)$. Its original and simplest form is the holomorphic functional calculus for a single operator $T \in \mathcal{B}(X)$, which can be expressed as a unital continuous algebra homomorphism $\Gamma: \mathcal{O}(U) \rightarrow \mathcal{B}(X)$ from the Fréchet algebra $\mathcal{O}(U)$ (furnished with the compact-open topology) of holomorphic functions on a domain $U \subseteq \mathbb{C}$ into the Banach algebra $\mathcal{B}(X)$ such that $\Gamma(z)=T$. The well known Gelfand-Dunford-Riesz theorem (see for instance [14, 2.2.15], $[15,8.2 .3])$ asserts that $\Gamma$ exists if and only if $\sigma(T) \subseteq U$, where $\sigma(T)$ is the usual spectrum of $T$.

The multivariable version of this result is of great interest and it has turned out to be more complicated. The statement of the problem is the

2000 Mathematics Subject Classification: Primary 47A60; Secondary 47A13, 17B30.

Key words and phrases: quasispectra, solvable Lie algebra, functional calculus, joint spectral radius. 
following. Let $T=\left(T_{1}, \ldots, T_{n}\right)$ be an operator family in $\mathcal{B}(X)$ and let $U$ be a domain in $\mathbb{C}^{n}$. Again by a holomorphic functional calculus on $U$ for $T$ we mean a continuous algebra homomorphism $\Gamma: \mathcal{O}(U) \rightarrow \mathcal{B}(X)$ such that $\Gamma\left(z_{i}\right)=T_{i}$ for all $i, 1 \leq i \leq n$. Since $\mathcal{O}(U)$ is a commutative algebra, it has a commutative homomorphic image, in particular, $T_{i} T_{j}=T_{j} T_{i}$ for all $i, j$. So, $T$ must be a mutually commuting operator family and holomorphic functional calculus makes sense only for such operator families. The problem has been solved in [21] by J. Taylor. The key role in this matter is played, as in the one variable case, by the (joint) spectrum $\sigma(T)$ of the operator family $T$, which is a nonempty compact subset in $\mathbb{C}^{n}$. More precisely, the (Taylor) spectrum $\sigma(T)$ is defined as the set of those $\lambda \in \mathbb{C}^{n}$ such that the Koszul complex $\operatorname{Kos}(X, T-\lambda)$ associated with the (commutative) operator family $T-\lambda=\left(T_{1}-\lambda_{1}, \ldots, T_{n}-\lambda_{n}\right)$ is not exact [20]. Taylor's theorem [20], [21] asserts that if $\sigma(T) \subseteq U$ then there is such a calculus $\Gamma$, and this condition is necessary if $U$ is a domain of holomorphy. Note that the Taylor spectrum has a complicated nature expressed in homological terms.

And what about an operator family $T$ which is not commutative? Obviously, in this case we cannot use the algebras $\mathcal{O}(U)$ to have a "noncommutative holomorphic functional calculus" for $T$. So, one has to change the function concept itself when developing a theory of functions in noncommuting variables. Here a whole range of new possibilities appear [22]. One of the approaches is based on the well developed Lie algebra technique (see [21, Section 7]). Namely, we fix a finite-dimensional Lie algebra $\mathfrak{g}$ instead of $\mathbb{C}^{n}$ and try to treat functions in elements of $\mathfrak{g}$ as elements of some topological completions of the universal enveloping (or polynomial) algebra $\mathcal{U}(\mathfrak{g})$. Evidently, the behavior of these completions depends of the variable space $\mathfrak{g}$. For instance, as observed in [21, Section 7], if the Lie algebra $\mathfrak{g}$ is "very" noncommutative, namely if it is a semisimple Lie algebra, then the algebra $\mathcal{O}_{\mathfrak{g}}$ (the Arens-Michael envelope [14, 5.2.21] of $\mathcal{U}(\mathfrak{g})$ ) of all entire functions in elements of $\mathfrak{g}$ is a topological direct product of full matrix algebras, that is, the structure of $\mathcal{O}_{\mathfrak{g}}$ is quite far from the one of usual entire functions in several complex variables. Moreover, in this case there is no reasonable functional calculus (see [21, Section 7]).

On the other hand, a satisfactory theory of holomorphic functions in noncommuting variables has been proposed for a solvable Lie algebra $\mathfrak{g}$ in [8], [9], [11]. Fix a finite-dimensional solvable Lie algebra $\mathfrak{g}$. Let $U$ be an absolutely convex domain in the dual space $\mathfrak{g}^{*}$ and let $\Delta(\mathfrak{g}) \subseteq \mathfrak{g}^{*}$ be the subspace of Lie characters, that is, those linear functionals on $\mathfrak{g}$ annihilating $[\mathfrak{g}, \mathfrak{g}]$. The Arens-Michael, Fréchet algebra $\mathcal{O}_{\mathfrak{g}}(U)$ of convergent power series or "holomorphic functions" on $U$ in elements of $\mathfrak{g}$ was introduced in [6], [8]. The noncommutative algebra $\mathcal{O}_{\mathfrak{g}}(U)$ is a Hausdorff, Fréchet, multinormed completion of the universal enveloping algebra $\mathcal{U}(\mathfrak{g})$ of $\mathfrak{g}$, so there 
is a canonical algebra homomorphism $\varphi: \mathcal{U}(\mathfrak{g}) \rightarrow \mathcal{O}_{\mathfrak{g}}(U)$ with dense range. Note that $\mathcal{O}_{\mathfrak{g}}(U)$ reduces to the algebra $\mathcal{O}(U)$ of usual holomorphic functions on $U$ in $n$ complex variables whenever $\mathfrak{g}=\mathbb{C}^{n}$ is a commutative Lie algebra $[8,4.2]$. In the general case, the algebra $\mathcal{O}_{\mathfrak{g}}(U)$ is commutative modulo its Jacobson radical $\operatorname{Rad} \mathcal{O}_{\mathfrak{g}}(U)$ and the quotient algebra $\mathcal{O}_{\mathfrak{g}}(U) / \operatorname{Rad} \mathcal{O}_{\mathfrak{g}}(U)$ is topologically isomorphic to $\mathcal{O}\left(U_{\mathrm{s}}\right)$ [8, Theorem 4.6], where $U_{\mathrm{s}}=U \cap \Delta(\mathfrak{g})$.

The functional calculus problem with respect to the "function" algebras $\mathcal{O}_{\mathfrak{g}}(U)$, where $U$ runs over all absolutely convex domains in $\mathfrak{g}^{*}$, is to determine a domain $U$ so that a given Lie algebra homomorphism $\pi: \mathfrak{g} \rightarrow A$ into a Banach algebra $A$ extends continuously to an algebra homomorphism $\widetilde{\pi}$ : $\mathcal{O}_{\mathfrak{g}}(U) \rightarrow A$, that is, $\tilde{\pi} \cdot \varphi=\pi$. The problem was solved only for a nilpotent Lie algebra $\mathfrak{g}$ in [8], in terms of Słodkowski spectra of an operator family $(A=\mathcal{B}(X))$. Namely, let $\pi: \mathfrak{g} \rightarrow \mathcal{B}(X)$ be a representation of a nilpotent Lie algebra $\mathfrak{g}$ on a Banach space $X$ and let $\sigma(\pi)$ be a Słodkowski spectrum of $\pi$ ([3]; see [18] for the commutative case), which is a compact subset in $\Delta(\mathfrak{g})$. Then $\pi$ has a continuous extension $\widetilde{\pi}: \mathcal{O}_{\mathfrak{g}}(U) \rightarrow \mathcal{B}(X)$ if and only if $\sigma(\pi) \subset U$. That is a version of (noncommutative) holomorphic functional calculus for absolutely convex domains.

A noncommutative spectral theory in the solvable Lie algebra framework started to develop independently of the functional calculus, in [3], [13], [16] and [7]. It was established that some properties of the commutative spectral theory remain true in the solvable Lie algebra case, but a comprehensive spectral theory remains to be developed in the nilpotent Lie algebra case.

How to introduce algebras $\mathcal{O}_{\mathfrak{g}}(U)$ for other domains $U$ and solve the relevant functional calculus problem? One of the possible approaches is based on homological ideas and Taylor's program [21]. These ideas were developed in [9]-[12] and the functional calculus problem was completely solved whenever $\pi(\mathfrak{g})$ is a supernilpotent Lie subalgebra in $\mathcal{B}(X)$, that is, $\pi([\mathfrak{g}, \mathfrak{g}])$ consists of nilpotent operators.

In contrast to serious advancements in the nilpotent case, almost nothing is known in the solvable Lie algebra case. The main property of the functional calculus, the spectral mapping theorem, remains inexplicit in this case. In this paper we propose a simple version of the functional calculus in the solvable Lie algebra case using the joint spectral radius technique. Namely, using the algebras $\mathcal{O}_{\mathfrak{g}}(U)$ when $U$ runs over all absolutely convex domains in the dual space $\mathfrak{g}^{*}$ of a solvable Lie algebra $\mathfrak{g}$, we propose a functional calculus in terms of quasispectra of a Lie algebra homomorphism into a Banach algebra, which extends the above mentioned result for the nilpotent case. A quasispectrum (see Definition 1) is a more general and simpler notion than Słodkowski (in particular, Taylor) spectrum, and it is not described in homological terms. Roughly speaking, a quasispectrum $\sigma(\pi)$ of a Lie homomorphism $\pi: \mathfrak{g} \rightarrow A$ into a Banach algebra $A$ is a compact subset 
in $\mathfrak{g}^{*}$ with the following property:

$$
\varrho(\pi(x))=\max \{|\lambda(x)|: \lambda \in \sigma(\pi)\}, \quad x \in \mathfrak{g},
$$

where $\varrho(\pi(x))$ is the spectral radius of $\pi(x)$ in $A$. Since a Słodkowski spectrum has the projection property [6], all Słodkowski spectra are clearly quasispectra. For other examples see Section 3 below. As the main result we prove that a Lie algebra homomorphism $\pi: \mathfrak{g} \rightarrow A$ of a solvable Lie algebra $\mathfrak{g}$ into a Banach algebra $A$ has a continuous extension $\tilde{\pi}: \mathcal{O}_{\mathfrak{g}}(U) \rightarrow A$ if and only if $\sigma(\pi) \subset U$ for a quasispectrum $\sigma(\pi)$ of $\pi$. In particular, we prove that the absolutely convex hulls of all quasispectra coincide and the joint spectral radius of a compact subset in a finite-dimensional solvable Lie subalgebra of a Banach algebra coincides with the geometric spectral radius calculated by means of an arbitrary quasispectrum (see Corollary 5). That is the geometrical characterization of the joint spectral radius.

I wish to thank Yu. V. Turovskir for useful discussions on the results of this note. I am also grateful to the referees for their attentive reading of the paper.

2. Preliminaries. All linear spaces considered are assumed to be complex and $\mathbb{C}$ denotes the field of complex numbers. The inner product in the Euclidean space $\mathbb{C}^{n}$ is denoted by $\langle\cdot, \cdot\rangle$, thus $\langle c, \lambda\rangle=\sum_{i=1}^{n} c_{i} \bar{\lambda}_{i}$ for vectors $c=\left(c_{1}, \ldots, c_{n}\right)$ and $\lambda=\left(\lambda_{1}, \ldots, \lambda_{n}\right)$ in $\mathbb{C}^{n}$, where $\bar{\lambda}_{i}$ is the complex conjugate of $\lambda_{i}, 1 \leq i \leq n$. In particular, $\|c\|=\langle c, c\rangle^{1 / 2}$ is the Euclidean norm of $c$. The set of all positive integers is denoted by $\mathbb{N}$, and $2^{S}$ is the set of all subsets of the set $S$. The unit ball of a normed space $X$ furnished with a norm $p$ is denoted by $B_{p}$, and $p^{*}$ denotes the dual norm on the dual space $X^{*}$. For a subset $M \subseteq X$, abc $M$ denotes the absolutely convex hull of $M$, that is, the set of all absolutely convex combinations $\sum_{i=1}^{n} c_{i} m_{i}$, $\sum\left|c_{i}\right| \leq 1, c_{i} \in \mathbb{C}, m_{i} \in M$. Consider a subset $F \subseteq X^{*}$ and a point $x \in X$. The subset $\{\lambda(x): \lambda \in F\} \subseteq \mathbb{C}$ is denoted by $F(x)$. The Banach space of all continuous complex functions on a compact topological space $K$, furnished with the uniform norm $\|f\|_{\infty}=\sup \{|f(x)|: x \in K\}$, is denoted by $\mathcal{C}(K)$, and the space of all holomorphic functions on an open subset $U \subseteq \mathbb{C}^{n}$, furnished with the compact-open topology, is denoted by $\mathcal{O}(U)$. The topological interior (respectively, boundary) of a subset $K$ in a topological space $\Omega$ is denoted by int $K$ (respectively, $\partial K$ ).

Let $A$ be a (non)associative algebra. An algebra homomorphism $\lambda: A$ $\rightarrow \mathbb{C}$ is said to be a character of $A$, and the set of all characters of $A$ is denoted by $\Delta(A)$. Let $A$ be a Banach (associative) algebra. We write $\operatorname{sp}_{A}(a)$ and $\varrho(a)$ for the spectrum and spectral radius of an element $a \in A$.

Now let $c=\left(c_{1}, \ldots, c_{n}\right) \in \mathbb{C}^{n}$ and let $a=\left(a_{1}, \ldots, a_{n}\right)$ be an $n$-tuple in a Banach algebra $A$. The symbol $\langle c, a\rangle_{A}$ for the linear combination $\sum_{i=1}^{n} c_{i} a_{i}$ 
in $A$ will be useful in Section 3. Note that if $a=\lambda=\left(\lambda_{1}, \ldots, \lambda_{n}\right)$ is a tuple in $\mathbb{C}$ then $\langle c, \lambda\rangle_{\mathbb{C}}=\langle c, \bar{\lambda}\rangle$ is the inner product of the vectors $c$ and $\bar{\lambda}=\left(\bar{\lambda}_{1}, \ldots, \bar{\lambda}_{n}\right)$ in $\mathbb{C}^{n}$.

2.1. The maximum formula for the joint spectral radius. Let $A$ be a Banach algebra and let $M \subseteq A$. We set $M^{n}=\left\{m_{1} \cdots m_{n}: m_{i} \in M\right\}$, $n \in \mathbb{N}$, and $\|M\|=\sup \{\|m\|: m \in M\}$, in particular, if $M \subseteq \mathbb{C}$ then $|M|=\sup \{|z|: z \in M\}$. The multiplicative semigroup in $A$ generated by $M$ is denoted by $\mathrm{SG}(M)$. By the very definition, $\operatorname{SG}(M)=\bigcup_{n \in \mathbb{N}} M^{n}$. If $M$ is bounded, that is, $\|M\|<\infty$, then the limit $\lim _{n}\left\|M^{n}\right\|^{1 / n}$ exists and equals $\inf _{n}\left\|M^{n}\right\|^{1 / n}$; we denote it by $\varrho|M|$ and call the joint spectral radius of $M$ [17]. (The conventional notation for the joint spectral radius is $\varrho(M)$, but we want to avoid misunderstanding it for the image of the set $M$ via the map $\varrho$.)

Recall the following maximum formula for the joint spectral radius proved by Yu. V. Turovskiı̌ and V. S. Shulman in [23, Theorem 9].

TheOREM 1. Let $A$ be a Banach algebra with a finite-dimensional solvable Lie subalgebra $\mathfrak{g}$ such that $A$ coincides with the closed associative subalgebra generated by $\mathfrak{g}$. Then $A$ is commutative modulo its Jacobson radical $\operatorname{Rad} A$ and

$$
\varrho|M|=|\varrho(M)|
$$

for any precompact subset $M \subseteq A$.

Note that this nontrivial result is based on the topological radical technique developed in [23].

REMARK 1. It is well known (see for instance [4, 1.3.3]) that the spectral radius is a continuous seminorm on a commutative Banach algebra. Moreover, if $A$ is a Banach algebra then $\operatorname{sp}_{A}(a)=\operatorname{sp}_{A / \operatorname{Rad} A}(\tau(a))$ and therefore $\varrho(a)=\varrho(\tau(a))$, where $\tau: A \rightarrow A / \operatorname{Rad} A$ is the quotient map. Hence $\varrho$ turns out to be a continuous seminorm on a Banach algebra commutative modulo $\operatorname{Rad} A$. Using Theorem 1, we conclude that

$$
\begin{aligned}
\varrho|M| & =|\varrho(M)|=\sup \{\varrho(m): m \in M\}=\sup \{\varrho(\tau(m)): m \in M\} \\
& =\max \{\varrho(\tau(m)): m \in M\}=\max \{\varrho(m): m \in M\},
\end{aligned}
$$

whenever $M$ is a compact subset in the closed associative envelope $A$ of $\mathfrak{g}$.

2.2. Banach enveloping algebras. Let $\mathfrak{g}$ be a finite-dimensional complex Lie algebra, $\mathcal{U}(\mathfrak{g})$ the universal enveloping algebra of $\mathfrak{g}$, and $p$ a norm on $\mathfrak{g}$. The Lie algebra $\mathfrak{g}$ furnished with the norm $p$ is a Banach-Lie algebra, hence one can define its Banach enveloping algebra $\mathcal{A}(p)$ [6], [8, Section 2.1]. The latter is a Hausdorff seminorm-completion of the enveloping algebra $\mathcal{U}(\mathfrak{g})$ with respect to a certain seminorm associated with $p$; consequently, there is a canonical algebra homomorphism $\varphi_{p}: \mathcal{U}(\mathfrak{g}) \rightarrow \mathcal{A}(p)$ with dense range. 
The following assertion proved in [6] characterizes the Banach enveloping algebras.

Lemma 1. Let $p$ be a norm on $\mathfrak{g}, A$ a Banach algebra, and $\pi: \mathfrak{g} \rightarrow A$ a Lie algebra homomorphism with the bounded semigroup $\operatorname{SG}\left(\pi\left(B_{p}\right)\right)$. There exists a unique bounded algebra homomorphism $\tilde{\pi}: \mathcal{A}(p) \rightarrow A$ such that $\widetilde{\pi} \cdot \varphi_{p}=\pi$.

REMARK 2. Note that $\mathrm{SG}\left(\pi\left(B_{p}\right)\right)$ is bounded whenever $\varrho\left|\pi\left(B_{p}\right)\right|<1$.

Now let $p$ and $q$ be norms on $\mathfrak{g}$ such that $p \leq q$. The canonical Lie algebra homomorphism $\varphi_{p}: \mathfrak{g} \rightarrow \mathcal{A}(p)$ has norm at most 1 whenever $\mathfrak{g}$ is furnished with the norm $q$. By Lemma 1 , there is a bounded algebra homomorphism $u: \mathcal{A}(q) \rightarrow \mathcal{A}(p)$, with $\|u\| \leq 1$, extending the identity map on $\mathcal{U}(\mathfrak{g})$.

2.3. Absolutely convex domains. The aim of this subsection is to demonstrate a connection between increasing norm sets on a finite-dimensional linear space and absolutely convex domains in its dual space.

Let $L$ be a finite-dimensional linear space. A norm set $p_{\Lambda}=\left\{p_{\alpha}\right\}_{\alpha \in \Lambda}$ defined on $L$ is said to be saturated if $p_{\Lambda}$ contains the maxima of all its finite subsets. A saturated norm set $p_{\Lambda}$ on $L$ is called increasing if for each $\alpha \in \Lambda$ there exists $\beta \in \Lambda$ with $p_{\alpha}(x)<p_{\beta}(x)$ for all $x \neq 0$ (we then write $p_{\alpha} \ll p_{\beta}$ ). Note that $p_{\alpha} \ll p_{\beta}$ iff $p_{\alpha} \leq \varepsilon_{\alpha \beta} p_{\beta}$, where $\varepsilon_{\alpha \beta}=\sup \left\{p_{\alpha}(x): x \in B_{p_{\beta}}\right\}<1$. Increasing norm sets $p_{\Lambda}=\left\{p_{\alpha}\right\}_{\alpha \in \Lambda}$ and $q_{\Omega}=\left\{q_{\gamma}\right\}_{\gamma \in \Omega}$ on $L$ are said to be equivalent $\left(p_{\Lambda} \sim q_{\Omega}\right)$ if for each $\alpha \in \Lambda$ there is a $\beta \in \Omega$ with $p_{\alpha} \ll q_{\beta}$ and vice versa $[8,2.2]$.

Let $M \subseteq L$ and $F \subseteq L^{*}$. The set $\widehat{M}_{F}=\{x \in L:|\lambda(x)| \leq|\lambda(M)|, \lambda \in F\}$ is called the $F$-convex hull of $M$ in $L$. A domain $U$ in $L$ is said to be $F$-convex if $\widehat{K}_{F} \subset U$ for each compact subset $K \subset U$ with $\operatorname{int} K \neq \emptyset$. Since $L^{* *}=L$, it makes sense to consider the $M$-convex hull $\widehat{F}_{M}$ of a subset $F \subseteq L^{*}$. For completeness, we present a detailed proof of the following assertion (see $[8$, Lemma 4.1]).

Lemma 2. Let $K$ be a compact subset in $L$ with $\operatorname{int} K \neq \emptyset$. Then $\widehat{K}_{L^{*}}=$ abc $K$.

Proof. By assumption $V_{\varepsilon}(x) \subset K$ for a certain open ball $V_{\varepsilon}(x)$ of radius $\varepsilon$ centered at $x \in \operatorname{int} K$. Then $V_{\varepsilon}(-x)=-V_{\varepsilon}(x) \subseteq$ abc $K$ and $V_{\varepsilon}(0) \subseteq$ $2^{-1} V_{\varepsilon}(x)+2^{-1} V_{\varepsilon}(-x) \subseteq$ abc $K$, so $0 \in \operatorname{int}$ abc $K$. Thus abc $K$ is a compact absorbing circled convex set, so its Minkowski functional $p$ is a norm. As abc $K$ is a closed set, we conclude that abc $K=B_{p}$.

Take $x \in \widehat{K}_{L^{*}}$. Then $|\lambda(x)| \leq|\lambda(K)|$ for all $\lambda \in L^{*}$. Since $K \subseteq B_{p}$, it follows that $|\lambda(x)| \leq\left|\lambda\left(B_{p}\right)\right|=p^{*}(\lambda)$. But $L^{* *}=L$ and $p^{* *}=p$, therefore $p(x) \leq 1$, that is, $x \in B_{p}=\operatorname{abc} K$. Therefore $\widehat{K}_{L^{*}} \subseteq$ abc $K$. 
Conversely, take $x=\sum a_{i} x_{i} \in \operatorname{abc} K$ with $x_{i} \in K, \sum\left|a_{i}\right| \leq 1$. Then $|\lambda(x)| \leq \sum\left|a_{i}\right|\left|\lambda\left(x_{i}\right)\right| \leq|\lambda(K)|, \lambda \in L^{*}$, whence $x \in \widehat{K}_{L^{*}}$. Thus abc $K$ $\subseteq \widehat{K}_{L^{*}}$.

Corollary 1. Let $U$ be a domain in $L$. Then $U$ is $L^{*}$-convex iff abc $K$ $\subset D$ for all compact subsets $K \subset D$, int $K \neq \emptyset$. Thus $U$ is $L^{*}$-convex iff $U$ is absolutely convex.

Now let $p_{\Lambda}=\left\{p_{\alpha}\right\}_{\alpha \in \Lambda}$ be an increasing norm set on $L$, and let $B_{\alpha}^{*}=B_{p_{\alpha}^{*}}$, $\alpha \in \Lambda$, be the unit balls in the dual space $L^{*}$. Assume that $p_{\alpha} \ll p_{\beta}$ and take $\lambda \in B_{\alpha}^{*}$. Then $p_{\beta}^{*}(\lambda)=\left|\lambda\left(B_{p_{\beta}}\right)\right| \leq \sup \left\{|\lambda(x)|: p_{\alpha}(x) \leq \varepsilon_{\alpha \beta}\right\} \leq \varepsilon_{\alpha \beta} p_{\alpha}^{*}(\lambda) \leq$ $\varepsilon_{\alpha \beta}<1$, that is, $\lambda \in \operatorname{int} B_{\beta}^{*}$. Thus $B_{\alpha}^{*} \subseteq$ int $B_{\beta}^{*}$ whenever $p_{\alpha} \ll p_{\beta}$. Put $U=\bigcup_{\alpha \in \Lambda} B_{\alpha}^{*}$. Then $U=\bigcup_{\alpha \in \Lambda}$ int $B_{\alpha}^{*}$ is a domain in $L^{*}$, for $p_{\Lambda}$ is an increasing norm set. We say that $U$ is the domain associated with the norm set $p_{\Lambda}$. By Corollary 1 , the domain $U$ associated with an increasing norm set $p_{\Lambda}$ is an absolutely convex domain in $L^{*}$. Obviously, equivalent norm sets have the same associated domain in $L^{*}$. Moreover, if $p_{\Lambda}$ and $q_{\Omega}$ are increasing norm sets with the same associated domain $U$ then these norm sets must be equivalent, that is, $p_{\Lambda} \sim q_{\Omega}$. Indeed, if $B_{\gamma}^{* \prime}$ is the unit ball in $L^{*}$ with respect to the dual norm $q_{\gamma}^{*}, \gamma \in \Omega$, then taking into account that $U=\bigcup_{\alpha \in \Lambda} B_{\alpha}^{*}=$ $\bigcup_{\gamma \in \Omega} B_{\gamma}^{* \prime}$ and $p_{\Lambda}$ (respectively, $q_{\Omega}$ ) is a saturated set, we conclude that $B_{\gamma}^{* \prime} \subseteq$ int $B_{\alpha}^{*}$ for some $\alpha \in \Lambda$. Further, by the maximum modulus principle, we deduce that $q_{\gamma}(x)=q_{\gamma}^{* *}(x)=\left|x\left(B_{\gamma}^{* \prime}\right)\right|<\left|x\left(B_{\alpha}^{*}\right)\right|=p_{\alpha}(x), x \neq 0$, that is, $q_{\gamma} \ll p_{\alpha}$. By analogy, for each $\alpha \in \Lambda$ there is $\gamma \in \Omega$ such that $p_{\alpha} \ll q_{\gamma}$, so $p_{\Lambda} \sim q_{\Omega}$. Thus the equivalence class $\left[p_{\Lambda}\right]$ of an increasing norm set $p_{\Lambda}$ has the same associated domain $U$. Moreover, if $U$ is an absolutely convex domain in $L^{*}$ then $U$ is associated with a certain increasing norm set $p_{\Lambda}$ on $L$ [8, Lemma 4.2]. Thus the following assertion is valid.

THEOREM 2. The correspondence $\left[p_{\Lambda}\right] \mapsto U$ is a bijection between the equivalence classes of increasing norm sets on $L$ and absolutely convex domains in $L^{*}$.

2.4. Holomorphic functions. Let $\mathfrak{g}$ be a finite-dimensional Lie algebra and let $U$ be an absolutely convex domain in $\mathfrak{g}^{*}$. By Theorem 2, the domain $U$ is associated with a unique (up to equivalence) increasing norm set $p_{\Lambda}=\left\{p_{\alpha}\right\}_{\alpha \in \Lambda}$ on $\mathfrak{g}$. Consider the Banach enveloping algebras $\mathcal{A}\left(p_{\alpha}\right)$ with $\alpha \in \Lambda$ (see Subsection 2.2). If $p_{\alpha} \ll p_{\beta}$ then we have a bounded algebra homomorphism $u_{\alpha \beta}: \mathcal{A}\left(p_{\beta}\right) \rightarrow \mathcal{A}\left(p_{\alpha}\right),\left\|u_{\alpha \beta}\right\| \leq 1$ (see Subsection $2.2)$ extending the identity map on the enveloping algebra $\mathcal{U}(\mathfrak{g})$. Moreover, $u_{\alpha \beta} \cdot u_{\beta \gamma}=u_{\alpha \gamma}$ whenever $p_{\alpha} \ll p_{\beta} \ll p_{\gamma}$, due to the uniqueness property asserted in Lemma 1. Thus, we have a projective system of Banach algebras $\mathfrak{A}\left(p_{\Lambda}\right)=\left\{\mathcal{A}\left(p_{\alpha}\right), u_{\alpha \beta}: \alpha, \beta \in \Lambda\right\}$; let $\lim _{\longleftarrow} \mathfrak{A}\left(p_{\Lambda}\right)$ be its inverse limit. Since the $\mathcal{A}\left(p_{\alpha}\right), \alpha \in \Lambda$, are associative algebras, $\lim _{\longleftarrow} \mathfrak{A}\left(p_{\Lambda}\right)$ inherits the alge- 
braic structure and it becomes a Fréchet, Arens-Michael algebra [14, 5.2.10]. Note that the algebras $\lim _{\mathfrak{A}} \mathfrak{A}\left(p_{\Lambda}\right)$ and $\lim \mathfrak{A}\left(q_{\Omega}\right)$ are topologically isomorphic whenever $p_{\Lambda} \sim q_{\Omega}$ [8, Lemma 2.6]. Thus $\varliminf_{\mathfrak{M}} \mathfrak{A}\left(p_{\Lambda}\right)$ depends only on the choice of the equivalence class $\left[p_{\Lambda}\right]$ or $U$ (see Theorem 2). Thus the Fréchet algebra $\mathcal{O}_{\mathfrak{g}}(U)=\lim \mathfrak{A}\left(p_{\Lambda}\right)$ with the canonical dense algebra homomorphism $\varphi: \mathcal{U}(\mathfrak{g}) \rightarrow \mathcal{O}_{\mathfrak{g}}(U)$ is well defined, and it is called an algebra of power series or holomorphic functions on $U$ in elements of $\mathfrak{g}$. The following assertion was proved in [8, Lemma 5.1].

Lemma 3. Let $\mathfrak{g}$ be a finite-dimensional Lie algebra, $U$ an absolutely convex domain in $\mathfrak{g}^{*}$ associated with a norm set $p_{\Lambda}=\left\{p_{\alpha}\right\}_{\alpha \in \Lambda}$, and $\pi$ : $\mathfrak{g} \rightarrow A$ a Lie homomorphism into a Banach algebra $A$. Then $\pi$ extends to a continuous algebra homomorphism $\tilde{\pi}: \mathcal{O}_{\mathfrak{g}}(U) \rightarrow A, \widetilde{\pi} \cdot \varphi=\pi$, iff $\varrho\left|\pi\left(B_{p_{\alpha}}\right)\right|<1$ for some $\alpha \in \Lambda$.

3. Quasispectra. In the following, $\mathfrak{g}$ denotes a finite-dimensional solvable Lie algebra. Let $\pi: \mathfrak{g} \rightarrow A$ and $\pi^{\prime}: \mathfrak{g} \rightarrow B$ be Lie homomorphisms into Banach algebras $A$ and $B$. These homomorphisms are understood to be equal, $\pi=\pi^{\prime}$, if $A=B$ and $\pi(x)=\pi^{\prime}(x)$ for all $x \in \mathfrak{g}$. Let us introduce the set $\mathfrak{B} \mathfrak{a}_{\mathfrak{g}}$ of all Lie homomorphisms of $\mathfrak{g}$ into Banach algebras and let $\mathfrak{B s}_{\mathfrak{g}}$ be its subset comprising all representations of $\mathfrak{g}$ on Banach spaces, that is, Lie homomorphisms like $\mathfrak{g} \rightarrow \mathcal{B}(X)$.

Definition 1. Let $\mathfrak{M}_{\mathfrak{g}} \subseteq \mathfrak{B a}_{\mathfrak{g}}$ be a subset. A map $\sigma: \mathfrak{M}_{\mathfrak{g}} \rightarrow 2^{\mathfrak{g}^{*}}$ is said to be a quasispectrum on $\mathfrak{g}$ with respect to $\mathfrak{M}_{\mathfrak{g}}$ if $\sigma(\pi)$ is a nonempty compact subset in $\mathfrak{g}^{*}$ and

$$
\varrho(\pi(x))=|\sigma(\pi)(x)|, \quad x \in \mathfrak{g},
$$

for each $\pi \in \mathfrak{M}_{\mathfrak{g}}$. The set of all quasispectra on $\mathfrak{g}$ with respect to the subset $\mathfrak{M}_{\mathfrak{g}}$ is denoted by $\mathfrak{Q}\left(\mathfrak{M}_{\mathfrak{g}}\right)$.

If $\mathfrak{g}$ is a Lie subalgebra of a certain Banach algebra $A$ then $\sigma(\mathfrak{g})(\sigma \in$ $\left.\mathfrak{Q}\left(\mathfrak{M}_{\mathfrak{g}}\right)\right)$ denotes the quasispectrum $\sigma\left(\mathrm{id}_{\mathfrak{g}}\right)$ of the identity homomorphism $\operatorname{id}_{\mathfrak{g}}: \mathfrak{g} \rightarrow A$.

Note that here we have followed the classical approach (see for instance [19], [5]) to determine a quasispectrum $\sigma$ as a set-valued function defined on Lie algebra homomorphisms into Banach algebras. In the commutative case it can be regarded as a set-valued function on finite commutative families in Banach algebras instead of Lie homomorphisms. Namely, assume that $\mathfrak{g}=\mathbb{C}^{n}$ is a commutative Lie algebra. Each Lie algebra homomorphism $\pi$ : $\mathbb{C}^{n} \rightarrow A$ is completely determined by the commutative family $a=\left(a_{1}, \ldots, a_{n}\right)$ in $A$, where $a_{i}=\pi\left(z_{i}\right), 1 \leq i \leq n$. So, one may use the notation $\sigma(a)$ instead of $\sigma(\pi)$ for a quasispectrum. Moreover, each linear functional $\lambda \in\left(\mathbb{C}^{n}\right)^{*}$ can be identified with a vector $\left(\lambda_{1}, \ldots, \lambda_{n}\right) \in \mathbb{C}^{n}$ such that $\lambda_{i}=\lambda\left(z_{i}\right)$ for all $i$. 
Using this identification and Definition 1, one can easily conclude that $\sigma(a)$ is a nonempty compact subset in $\mathbb{C}^{n}$ with the property

$$
\varrho\left(\langle c, a\rangle_{A}\right)=\max \{|\langle c, \bar{\lambda}\rangle|: \lambda \in \sigma(a)\}=|\langle c, \overline{\sigma(a)}\rangle|
$$

for all $c=\left(c_{1}, \ldots, c_{n}\right) \in \mathbb{C}^{n}$, where $\langle c, a\rangle_{A}=\sum_{i=1}^{n} c_{i} a_{i} \in A$. Moreover, in (3.1) it suffices to consider only those vectors $c \in \mathbb{C}^{n}$ with $\sum_{i=1}^{n}\left|c_{i}\right| \leq 1$. In particular,

$$
\varrho\left(a_{i}\right)=\max \left\{\left|\lambda_{i}\right|: \lambda=\left(\lambda_{1}, \ldots, \lambda_{n}\right) \in \sigma(a)\right\}
$$

for all $i$.

LEMMA 4. Let $\sigma(\pi)$ be a quasispectrum of a Lie homomorphism $\pi: \mathfrak{g} \rightarrow$ $A$ of $\mathfrak{g}$ into a Banach algebra $A$. Then $\sigma(\pi) \subseteq \Delta(\mathfrak{g})$.

Proof. Note that $\pi(\mathfrak{g})$ is a solvable Lie subalgebra in $A$ and the closed associative subalgebra $B$ in $A$ generated by $\pi(\mathfrak{g})$ is commutative modulo its Jacobson radical $\operatorname{Rad} B$ by Theorem 1 , whence $\pi([\mathfrak{g}, \mathfrak{g}])=[\pi(\mathfrak{g}), \pi(\mathfrak{g})] \subseteq$ $\operatorname{Rad} B$. Thus $\varrho(\pi(x))=0$ for all $x \in[\mathfrak{g}, \mathfrak{g}]$. By Definition $1,|\lambda(x)| \leq \varrho(\pi(x))$ for all $\lambda \in \sigma(\pi)$, therefore $\lambda(x)=0$ for $\lambda \in \sigma(\pi)$, that is, $\lambda([\mathfrak{g}, \mathfrak{g}])=0$. Hence $\lambda \in \Delta(\mathfrak{g})$.

Let us provide some examples of quasispectra.

Proposition 1. Let $\mathfrak{M}_{\mathfrak{g}}$ be a set of Lie homomorphisms of $\mathfrak{g}$ into $B a$ nach algebras $\left(\mathfrak{M}_{\mathfrak{g}} \subseteq \mathfrak{B a}_{\mathfrak{g}}\right)$ and let $\sigma: \mathfrak{M}_{\mathfrak{g}} \rightarrow 2^{\mathfrak{g}^{*}}$ be a map such that $\sigma(\pi)$ is a nonempty compact subset in $\mathfrak{g}^{*}$ and

$$
\partial \operatorname{sp}(\pi(x)) \subseteq \sigma(\pi)(x) \subseteq \operatorname{sp}(\pi(x))
$$

for all $x \in \mathfrak{g}$. Then $\sigma$ is a quasispectrum on $\mathfrak{M}_{\mathfrak{g}}$, that is, $\sigma \in \mathfrak{Q}\left(\mathfrak{M}_{\mathfrak{g}}\right)$. In particular, all Cartan-Stodkowski (and Cartan-Taylor) spectra $\Sigma \in \mathfrak{K S}$ ([1], [11]) are quasispectra on $\mathfrak{g}$ with respect to the set $\mathfrak{B s}_{\mathfrak{g}}$ of all Banach space representations of $\mathfrak{g}$, that is, $\mathfrak{K} \mathfrak{S} \subseteq \mathfrak{Q}\left(\mathfrak{B s}_{\mathfrak{g}}\right)$. Moreover, if $\Sigma$ is the CartanTaylor spectrum then $\sigma=\partial \Sigma$ defined by $\sigma(\pi)=\partial \Sigma(\pi)$ is a quasispectrum.

Proof. Using the inclusions $\partial \operatorname{sp}(\pi(x)) \subseteq \sigma(\pi)(x) \subseteq \operatorname{sp}(\pi(x))$, we deduce that

$$
\varrho(\pi(x))=\max \{|z|: z \in \partial \operatorname{sp}(\pi(x))\}=\max \{|\lambda(x)|: \lambda \in \sigma(\pi)\}=|\sigma(\pi)(x)| .
$$

By Definition $1, \sigma \in \mathfrak{Q}\left(\mathfrak{M}_{\mathfrak{g}}\right)$.

Now let $\mathfrak{M}_{\mathfrak{g}}=\mathfrak{B S}_{\mathfrak{g}}$ and let $\Sigma \in \mathfrak{K} \mathfrak{S}$ be a Cartan-Słodkowski spectrum. Then

$$
\Sigma(\pi)(x)=\Sigma(\pi(x)) \subseteq \operatorname{sp}(\pi(x))
$$

due to the projection property [1], [11]. Moreover, $\partial \operatorname{sp}(\pi(x)) \subseteq \sigma_{\text {ap }}(\pi(x)) \subseteq$ $\Sigma(\pi(x))$, where $\sigma_{\text {ap }}(\pi(x))$ is the approximate point spectrum of the operator $\pi(x)$. Consequently, $\Sigma \in \mathfrak{Q}\left(\mathfrak{B s}_{\mathfrak{g}}\right)$. 
Finally, assume that $\Sigma$ is the Cartan-Taylor spectrum [1] and let $\sigma$ : $\mathfrak{B s}_{\mathfrak{g}} \rightarrow 2^{\mathfrak{g}^{*}}, \sigma(\pi)=\partial \Sigma(\pi)$. Note that $\operatorname{sp}(\pi(x))=\Sigma(\pi)(x)$ (see [1]). Bearing in mind that the projection map $\mathfrak{g}^{*} \rightarrow \mathbb{C}, \lambda \mapsto \lambda(x)$, is open, we conclude that $\partial \operatorname{sp}(\pi(x)) \subseteq \sigma(\pi)(x)$. It follows that $\sigma \in \mathfrak{Q}\left(\mathfrak{B s}_{\mathfrak{g}}\right)$.

Now let $\mathfrak{g}=\mathbb{C}^{n}$ be a commutative Lie algebra. For a commutative $n$ tuple $a=\left(a_{1}, \ldots, a_{n}\right)$ in a Banach algebra $A$, let $\sigma(a) \subset \mathbb{C}^{n}$ be a nonempty compact subset with the property

$$
\partial \operatorname{sp}\left(\langle c, a\rangle_{A}\right) \subseteq\{|\langle c, \bar{\lambda}\rangle|: \lambda \in \sigma(a)\} \subseteq \operatorname{sp}\left(\langle c, a\rangle_{A}\right)
$$

for all $c \in \mathbb{C}^{n}$ with $\sum_{i=1}^{n}\left|c_{i}\right| \leq 1$. By Proposition $1, \sigma$ is a quasispectrum. In particular, $\sigma(a) \subseteq \prod_{i=1}^{n} \operatorname{sp}\left(a_{i}\right)$.

Conversely, let $\sigma$ be any set-valued function defined on finite commutative tuples in Banach algebras with the properties:

(i) $\sigma(a) \subset \mathbb{C}^{n}$ is a compact nonempty subset for a commutative $n$ tuple $a$;

(ii) $\partial \operatorname{sp}(a) \subseteq \sigma(a) \subseteq \operatorname{sp}(a)$ for a singleton $a$;

(iii) $\sigma\left(\langle c, a\rangle_{A}\right)=\langle c, \overline{\sigma(a)}\rangle$ for all $c \in \mathbb{C}^{n}$ with $\sum_{i=1}^{n}\left|c_{i}\right| \leq 1$, and any commutative $n$-tuple $a$.

Then $\sigma$ is a quasispectrum due to Proposition 1. Note that axiom (iii) is a simple version of the polynomial mapping property, that is, $\sigma(p(a))=$ $p(\sigma(a))$ for all polynomial families $p$ in $n$ complex variables. It is well known [18] that all Słodkowski spectra $\sigma_{\pi, k}, \sigma_{\delta, k}, k \geq 0$, have the polynomial mapping property. Using it, we conclude that they are quasispectra with respect to the bounded linear operators. Indeed, it suffices (see [18]) to verify (ii) only. But this is evident from the inclusions

$$
\partial \operatorname{sp}(a) \subseteq \sigma_{\mathrm{ap}}(a)=\sigma_{\pi, 0}(a) \subseteq \sigma_{\pi, k}(a) \subseteq \operatorname{sp}(a)
$$

and

$$
\partial \operatorname{sp}(a)=\partial \operatorname{sp}\left(a^{*}\right) \subseteq \sigma_{\text {ap }}\left(a^{*}\right)=\sigma_{\pi, 0}\left(a^{*}\right)=\sigma_{\delta, 0}(a) \subseteq \sigma_{\delta, k}(a) \subseteq \operatorname{sp}(a)
$$

for an operator $a$ and for all $k \geq 0$.

Finally, note that the topological boundary of the Taylor spectrum satisfies axioms (i)-(iii), therefore it is a quasispectrum.

Now let us demonstrate that the notion of quasispectrum is more general than the one of joint spectrum. Let $\pi: \mathfrak{g} \rightarrow A$ be a representation of $\mathfrak{g}$ into a Banach algebra $A, B$ the closure of the associative envelope of $\pi(\mathfrak{g})$ in $A$, and $S^{n}$ the unit sphere with respect to the Euclidean norm $\|\cdot\|$ on $\mathfrak{g}$. The spectral radius $\varrho$ is a continuous seminorm on $B$ (see Remark 1). It follows that $N_{S^{n}}=\varrho\left(\pi\left(S^{n}\right)\right)$ is a compact subset of the real line and therefore $\left|N_{S^{n}}\right|<\infty$. Further, there is a functional $\lambda_{x} \in \mathfrak{g}^{*}$ with $\left|\lambda_{x}(y)\right| \leq \varrho(\pi(y))$ for all $y \in \mathfrak{g}$, and $\lambda_{x}(x)=\varrho(\pi(x))$ for each $x \in S^{n}$, thanks to the Hahn-Banach 
theorem. Then

$$
\left\|\lambda_{x}\right\|=\left|\lambda_{x}\left(S^{n}\right)\right| \leq\left|N_{S^{n}}\right|
$$

for all $x \in S^{n}$. Consequently, $\left\{\lambda_{x}: x \in S^{n}\right\}$ is a bounded set in $\mathfrak{g}^{*}$; let $\sigma(\pi)$ be its closure.

Proposition 2. The assignment $\pi \mapsto \sigma(\pi)$ is a quasispectrum on $\mathfrak{g}$ with respect to $\mathfrak{B} \mathfrak{a}_{\mathfrak{g}}$.

Proof. Clearly, $\sigma(\pi)$ is a compact subset in $\mathfrak{g}^{*}$. By Definition 1 , we have to prove that $\varrho(\pi(y))=|\sigma(\pi)(y)|$ for all $y \in \mathfrak{g}$. One can assume that $y \neq 0$, as otherwise the assertion is obvious. Take $\mu \in \sigma(\pi)$. Since $\sigma(\pi)$ is the closure of $\left\{\lambda_{x}: x \in S^{n}\right\}$, it follows that $|\mu(y)| \leq \varrho(\pi(y))$ for $y \in \mathfrak{g}$, whence $|\sigma(\pi)(y)| \leq \varrho(\pi(y))$. Moreover,

$$
\varrho(\pi(y))=\|y\| \varrho(\pi(y /\|y\|))=\|y\| \lambda_{y /\|y\|}(y /\|y\|)=\lambda_{y /\|y\|}(y) .
$$

Taking into account that $\lambda_{y /\|y\|} \in \sigma(\pi)$, we infer $|\sigma(\pi)(y)|=\varrho(\pi(y))$. Thus $\sigma \in \mathfrak{Q}\left(\mathfrak{B a} \mathfrak{a}_{\mathfrak{g}}\right)$.

Let us describe the latter quasispectrum in the commutative case. Fix a commutative $n$-tuple in a Banach algebra $A$. By construction, to each $c \in S^{n}$ there corresponds $c_{*} \in \mathbb{C}^{n}$ such that

$$
\left|\left\langle c, \bar{c}_{*}\right\rangle\right|=\varrho\left(\langle c, a\rangle_{A}\right) \quad \text { and } \quad\left|\left\langle t, \bar{c}_{*}\right\rangle\right| \leq \varrho\left(\langle t, a\rangle_{A}\right)
$$

for all $t \in \mathbb{C}^{n}$. The set $\left\{c_{*}: c \in S^{n}\right\}$ is bounded in $\mathbb{C}^{n}$, therefore it has a compact closure denoted by $\sigma(a)$, which is a quasispectrum of $a$, as proved in Proposition 2.

4. The functional calculus. Now we prove the main result of this paper on functional calculus.

THEOREM 3. Let $\mathfrak{g}$ be a finite-dimensional solvable Lie algebra, $\mathfrak{M}_{\mathfrak{g}} \subseteq$ $\mathfrak{B a}_{\mathfrak{g}}$ be a subset of Lie homomorphisms of $\mathfrak{g}$ into Banach algebras, $\pi: \mathfrak{g} \rightarrow A$ a Lie homomorphism from $\mathfrak{M}_{\mathfrak{g}}, U$ an absolutely convex domain in $\mathfrak{g}^{*}$, and $\sigma \in \mathfrak{Q}\left(\mathfrak{M}_{\mathfrak{g}}\right)$. Then $\pi$ extends to a continuous algebra homomorphism $\tilde{\pi}$ : $\mathcal{O}_{\mathfrak{g}}(U) \rightarrow A, \tilde{\pi} \cdot \varphi=\pi$, iff $\sigma(\pi) \subset U$.

Proof. By Theorem 2, the domain $U$ is associated with a norm set $p_{\Lambda}=$ $\left\{p_{\alpha}\right\}_{\alpha \in \Lambda}$ on the Lie algebra $\mathfrak{g}$. Let $B_{p_{\alpha}}$ be the unit ball in $\mathfrak{g}$ with respect to the norm $p_{\alpha}$. If the continuous algebra homomorphism $\tilde{\pi}: \mathcal{O}_{\mathfrak{g}}(U) \rightarrow A$ exists then $\varrho\left|\pi\left(B_{p_{\alpha}}\right)\right|<1$ for some $\alpha \in \Lambda$, by Lemma 3. Take $\lambda \in \sigma(\pi) \subseteq \Delta(\mathfrak{g})$ (Lemma 4). If $x \in B_{p_{\alpha}}$ then, by Definition 1 , we infer

$$
|\lambda(x)| \leq \varrho(\pi(x)) \leq \varrho\left|\pi\left(B_{p_{\alpha}}\right)\right|
$$

Therefore $p_{\alpha}^{*}(\lambda)=\left|\lambda\left(B_{p_{\alpha}}\right)\right|<1$. Thus $\lambda \in B_{\alpha}^{*}$, where $B_{\alpha}^{*}$ is the unit ball 
with respect to the norm $p_{\alpha}^{*}$. But $U=\bigcup_{\alpha \in \Lambda} B_{\alpha}^{*}$ (see Subsection 2.3), so $\lambda \in U$. Thus $\sigma(\pi) \subset U$.

Conversely, assume that $\sigma(\pi) \subset U$. Note that $\sigma(\pi) \subset \operatorname{int} B_{\alpha}^{*}$ for some $\alpha \in \Lambda$, for $U=\bigcup_{\alpha \in \Lambda}$ int $B_{\alpha}^{*}, \sigma(\pi)$ is a compact set by Definition 1 , and $p_{\Lambda}$ is an increasing norm set. Further, by Lemma 3 , it suffices to prove that $\varrho\left|\pi\left(B_{p_{\alpha}}\right)\right|<1$. Take $x \in B_{p_{\alpha}}$. By Definition 1 and the maximum modulus principle, we deduce that

$$
\begin{aligned}
\varrho(\pi(x)) & =\max \{|\lambda(x)|: \lambda \in \sigma(\pi)\}<\max \left\{|\lambda(x)|: \lambda \in B_{\alpha}^{*}\right\} \\
& \leq \max \left\{p_{\alpha}^{*}(\lambda) p_{\alpha}(x): \lambda \in B_{\alpha}^{*}\right\} \leq p_{\alpha}(x) \leq 1,
\end{aligned}
$$

that is, $\varrho(\pi(x))<1$. But $\varrho\left|\pi\left(B_{p_{\alpha}}\right)\right|=\left|\varrho\left(\pi\left(B_{p_{\alpha}}\right)\right)\right|=\max \left\{\varrho(y): y \in \pi\left(B_{p_{\alpha}}\right)\right\}$ by Theorem 1 and Remark 1. Consequently, $\varrho\left|\pi\left(B_{p_{\alpha}}\right)\right|<1$.

Corollary 2. Let $a=\left(a_{1}, \ldots, a_{n}\right)$ be a commutative $n$-tuple in a $B a$ nach algebra $A, U$ an absolutely convex domain in $\mathbb{C}^{n}$, and $\sigma(a)$ a quasispectrum of $a$. There is a continuous algebra homomorphism $\Gamma: \mathcal{O}(U) \rightarrow A$ (holomorphic functional calculus) such that $\Gamma\left(z_{i}\right)=a_{i}, 1 \leq i \leq n$, if and only if $\sigma(a) \subset U$. Then

CoRollary 3. Let $\mathfrak{g}$ be a solvable Lie algebra and let $\sigma_{1}, \sigma_{2} \in \mathfrak{Q}_{\mathfrak{g}}\left(\mathfrak{M}_{\mathfrak{g}}\right)$.

$$
\operatorname{abc} \sigma_{1}(\pi)=\operatorname{abc} \sigma_{2}(\pi)
$$

for all $\pi \in \mathfrak{M}_{\mathfrak{g}}$. In particular, abc $\sigma_{1}(a)=\operatorname{abc} \sigma_{2}(a)$ for any commutative tuple $a$ and quasispectra $\sigma_{1}, \sigma_{2}$.

Proof. Let $\pi: \mathfrak{g} \rightarrow A$ be a Lie homomorphism from $\mathfrak{M}_{\mathfrak{g}}$. By Definition $1, \sigma_{2}(\pi)$ is a compact set. If $U$ is an absolutely convex domain such that abc $\sigma_{2}(\pi) \subset U$ then using Theorem 3 we conclude that $\pi$ extends to a continuous algebra homomorphism $\tilde{\pi}: \mathcal{O}_{\mathfrak{g}}(U) \rightarrow A$. According again to Theorem 3, now with respect to the quasispectrum $\sigma_{1}$, we infer that $\sigma_{1}(\pi) \subseteq U$. Thus $\sigma_{1}(\pi) \subseteq$ abc $\sigma_{2}(\pi)$. Similarly, $\sigma_{2}(\pi) \subseteq$ abc $\sigma_{1}(\pi)$. Consequently, abc $\sigma_{1}(\pi)=\operatorname{abc} \sigma_{2}(\pi)$.

Corollary 3 is a noncommutative version of Theorem 8 in [5].

CoROLlary 4. Let $\mathfrak{g}$ be a solvable Lie algebra and let $\sigma_{1}, \sigma_{2} \in \mathfrak{Q}_{\mathfrak{g}}\left(\mathfrak{M}_{\mathfrak{g}}\right)$. If $q$ is a seminorm on $\mathfrak{g}^{*}$ then

$$
q\left(\sigma_{1}(\pi)\right)=q\left(\sigma_{2}(\pi)\right)
$$

In particular, if a is a commutative n-tuple in a Banach algebra then

$$
\|\sigma(a)\|=\max \left\{\left\|c_{*}\right\|: c \in S^{n}\right\}
$$

where $\|\cdot\|$ is the Euclidean norm in $\mathbb{C}^{n}$, and $c_{*} \in \mathbb{C}^{n}$ corresponds to $c \in S^{n}$ with the property (3.3). 
Proof. Using Corollary 3, we infer that

$$
q\left(\sigma_{1}(\pi)\right)=q\left(\operatorname{abc} \sigma_{1}(\pi)\right)=q\left(\operatorname{abc} \sigma_{2}(\pi)\right)=q\left(\sigma_{2}(\pi)\right),
$$

that is, $q\left(\sigma_{1}(\pi)\right)=q\left(\sigma_{2}(\pi)\right)$.

5. The geometric spectral radius. In this section we demonstrate that the joint spectral radius of a compact subset in a finite-dimensional solvable Lie subalgebra of a Banach algebra can be calculated by means of quasispectra.

Let again $\mathfrak{g}$ be a finite-dimensional solvable Lie algebra, $\mathfrak{M}_{\mathfrak{g}} \subseteq \mathfrak{B a}_{\mathfrak{g}}$ a subset of Lie homomorphisms of $\mathfrak{g}$ into Banach algebras, $\pi: \mathfrak{g} \rightarrow A$ a Lie homomorphism from $\mathfrak{M}_{\mathfrak{g}}$, and $\sigma \in \mathfrak{Q}\left(\mathfrak{M}_{\mathfrak{g}}\right)$. Consider a continuous map $\gamma$ : $\Omega \rightarrow \mathfrak{g}$ acting from a compact topological space $\Omega$ into $\mathfrak{g}$. We define a quasispectrum of the map $\pi \gamma: \Omega \rightarrow \mathfrak{g}$ by setting

$$
\sigma(\pi \gamma)=\{\lambda \gamma: \lambda \in \sigma(\pi)\}
$$

Obviously, $\sigma(\pi \gamma)$ is a subset of the Banach space $\mathcal{C}(\Omega)$, so one can define the geometric spectral radius of the map $\pi \gamma$ as in [2], by setting

$$
r_{\sigma, \infty}(\pi \gamma)=\|\sigma(\pi \gamma)\|_{\infty}
$$

Let $q_{\gamma}$ be the seminorm on $\mathfrak{g}^{*}$ given by $q_{\gamma}(\lambda)=\|\lambda \gamma\|_{\infty}$. Then $r_{\sigma, \infty}(\pi \gamma)=$ $q_{\gamma}(\sigma(\pi))$. Using Corollary 4 , we obtain

$$
r_{\sigma_{1}, \infty}(\pi \gamma)=r_{\sigma_{2}, \infty}(\pi \gamma)
$$

for all $\sigma_{1}, \sigma_{2} \in \mathfrak{Q}\left(\mathfrak{M}_{\mathfrak{g}}\right)$. Thus the geometric spectral radius does not depend upon the quasispectrum $\sigma \in \mathfrak{Q}\left(\mathfrak{M}_{\mathfrak{g}}\right)$. Therefore we set $r_{\infty}(\pi \gamma)=r_{\sigma, \infty}(\pi \gamma)$. The following assertion exhibits a connection between the geometric and joint spectral radii.

Proposition 3. $r_{\infty}(\pi \gamma)=|\varrho(\pi \gamma(\Omega))|=\varrho|\pi \gamma(\Omega)|$.

Proof. By using (5.1) and Definition 1, we deduce that

$$
\begin{aligned}
r_{\infty}(\pi \gamma) & =\sup \left\{\|\lambda \gamma\|_{\infty}: \lambda \in \sigma(\pi)\right\}=\sup _{\lambda \in \sigma(\pi)} \sup _{x \in \gamma(\Omega)}|\lambda(x)|=\sup _{x \in \gamma(\Omega)} \sup _{\lambda \in \sigma(\pi)}|\lambda(x)| \\
& =\sup _{x \in \gamma(\Omega)}|\sigma(\pi)(x)|=\sup _{x \in \gamma(\Omega)} \varrho(\pi(x))=|\varrho(\pi \gamma(\Omega))| .
\end{aligned}
$$

But $|\varrho(\pi \gamma(\Omega))|=\varrho|\pi \gamma(\Omega)|$ by Theorem 1 .

Note that Proposition 3 was proved by D. Beltiţa in [2, Theorem 4.1(2)] when $A=B(\mathfrak{X}), \pi(\mathfrak{g})$ consists of compact operators and $\sigma=\Sigma$ is the Cartan-Taylor spectrum (see Proposition 1).

Corollary 5. Let $A$ be a Banach algebra, $\mathfrak{g}$ a finite-dimensional solvable Lie subalgebra in $A$, and $M$ a compact subset in $\mathfrak{g}$. If $\sigma(\mathfrak{g})$ a quasispectrum of $\mathfrak{g}$ then

$$
\varrho|M|=\max \{|\lambda(M)|: \lambda \in \sigma(\mathfrak{g})\} .
$$


In particular, if $M=a$ is a commutative $n$-tuple in a Banach algebra then the above identity reduces to the maximum formula

$$
\varrho|a|=\max \left\{\varrho\left(a_{i}\right): 1 \leq i \leq n\right\} .
$$

Proof. It suffices to apply Proposition 3 by assuming that $\Omega=M$ and $\gamma$ is the identity map. Note also that $\left\|\left.\lambda\right|_{M}\right\|_{\infty}=|\lambda(M)|$ for all $\lambda \in \mathfrak{g}^{*}$.

Now let $a$ be a commutative $n$-tuple. Using (3.2), we deduce that

$$
\varrho|a|=\max _{\lambda \in \sigma(a)}|\lambda(a)|=\max _{\lambda \in \sigma(a)} \max _{i}\left|\lambda_{i}\right|=\max _{i} \max _{\lambda \in \sigma(a)}\left|\lambda_{i}\right|=\max _{i} \varrho\left(a_{i}\right),
$$

that is, $\varrho|a|=\max _{i} \varrho\left(a_{i}\right)$.

Finally, using Proposition 2 and Corollary 5, we conclude that

$$
\varrho|M|=\sup \left\{\left|\lambda_{x}(M)\right|: x \in S\right\},
$$

where $S$ is the unit sphere in $\mathfrak{g}$.

\section{References}

[1] D. Beltiţa, Spectrum for a solvable Lie algebra of operators, Studia Math. 135 (1999), 163-178.

[2] -, Analytic joint spectral radius in a solvable Lie algebra of operators, ibid. 144 (2001) 153-167.

[3] E. Boasso, Dual properties and joint spectra for solvable Lie algebras of operators, J. Operator Theory 33 (1995), 105-116.

[4] N. Bourbaki, Théories Spectrales, Hermann, 1967.

[5] M. Chō and W. Żelazko, On geometric spectral radius of commuting $n$-tuples of operators, Hokkaido Math. J. 21 (1992), 251-258.

[6] A. A. Dosiev, Holomorphic functions of a basis of a nilpotent Lie algebra, Funct. Anal. Appl. 34 (2000), 302-304.

[7] -, Spectra of parametrized Banach complexes, J. Operator Theory 48 (2002), 585614.

[8] -, Algebras of power series in elements of a Lie algebra and Stodkowski spectra, J. Math. Sci. 124 (2004), 4886-4908.

[9] - Homological dimensions of the algebra of entire functions of elements of a nilpotent Lie algebra, Funct. Anal. Appl. 37 (2003), 61-64.

[10] - Spectral mapping framework, in: Banach Center Publ. 67, Inst. Math., Polish Acad. Sci., 2005, 161-169.

[11] - Cartan-Stodkowski spectra, splitting elements and noncommutative spectral mapping theorems, J. Funct. Anal. 230 (2006), 446-493.

[12] - Cohomology of sheaves of Fréchet algebras and spectral theory, Funct. Anal. Appl. 39 (2005), 225-228.

[13] A. S. Fainshtein, Taylor joint spectrum for families of operators generating nilpotent Lie algebras, J. Operator Theory 29 (1993), 3-27; Corrections, ibid. 44 (2000), 433434.

[14] A. Ya. Helemskii, Banach and Polynormed Algebras: General Theory, Representations, Homology, Nauka, Moscow, 1989 (in Russian).

[15] S. S. Kutateladze, Fundamentals of Functional Analysis, Kluwer, 1996. 
[16] C. Ott, Gemeinsame Specktren für auflösbare Operator-Lie Algebren, Ph.D. thesis, Kiel, 1997 (http://analysis.math.uni-kiel.de/wrobel/).

[17] G.-C. Rota and W. G. Strang, A note on the joint spectral radius, Indag. Math. 22 (1960), 379-381.

[18] Z. Słodkowski, An infinite family of joint spectra, Studia Math. 61 (1977), 239-255.

[19] Z. Słodkowski and W. Żelazko, On joint spectra of commuting families of operators, ibid. 50 (1974), 127-148.

[20] J. L. Taylor, The analytic-functional calculus for several commuting operators, Acta Math. 125 (1970), 1-38.

[21] —, A general framework for a multi-operator functional calculus, Adv. Math. 9 (1972), 183-252.

[22] - Functions of several noncommuting variables, Bull. Amer. Math. Soc. 79 (1973), 1-34.

[23] Yu. V. Turovskiu and V. S. Shulman, Radicals in Banach algebras and some problems in the theory of radical Banach algebras, Funktsional. Anal. i Prilozhen. 35 (2001), no. 4, 88-91 (in Russian).

Department of Mathematics

Atilim University

Incek 06836

Ankara, Turkey

E-mail: adosiev@atilim.edu.tr dosiev@yahoo.com 